\title{
HOMOGENIZATION OF A SPECTRAL EQUATION WITH DRIFT IN LINEAR TRANSPORT
}

\author{
Guillaume BaL ${ }^{1}$
}

\begin{abstract}
This paper deals with the homogenization of a spectral equation posed in a periodic domain in linear transport theory. The particle density at equilibrium is given by the unique normalized positive eigenvector of this spectral equation. The corresponding eigenvalue indicates the amount of particle creation necessary to reach this equilibrium. When the physical parameters satisfy some symmetry conditions, it is known that the eigenvectors of this equation can be approximated by the product of two term. The first one solves a local transport spectral equation posed in the periodicity cell and the second one a homogeneous spectral diffusion equation posed in the entire domain. This paper addresses the case where these symmetry conditions are not fulfilled. We show that the factorization remains valid with the diffusion equation replaced by a convection-diffusion equation with large drift. The asymptotic limit of the leading eigenvalue is also modified. The spectral equation treated in this paper can model the stability of nuclear reactor cores and describe the distribution of neutrons at equilibrium. The same techniques can also be applied to the time-dependent linear transport equation with drift, which appears in radiative transfer theory and which models the propagation of acoustic, electromagnetic, and elastic waves in heterogeneous media.
\end{abstract}

Mathematics Subject Classification. 35B27, 35F05.

Received December 4, 2000. Revised May 22, 2001.

\section{INTRODUCTION}

The power distribution in a nuclear reactor is determined by solving a linear transport spectral equation for the neutron density of in the core. This equation expresses the balance between the production of neutrons by fission and its absorption in the reactor core and leakage at the boundary. The unknowns are the phase-space neutron density $\phi(x, v)$, where $x$ stands for position and $v$ for velocity, and the constant $k_{\text {eff }}$, which measures how much fission is necessary to reach an equilibrium. The couple $\left(k_{\mathrm{eff}}, \phi(x, v)\right)$ is the largest eigenvalue and the corresponding positive eigenvector of the equation

$$
\begin{aligned}
& v \cdot \nabla \phi+\Sigma(x, v) \phi=\int_{V} f\left(x, v^{\prime}, v\right) \phi\left(x, v^{\prime}\right) \mathrm{d} v^{\prime}+\frac{1}{k_{\text {eff }}} \int_{V} \sigma\left(x, v^{\prime}, v\right) \phi\left(x, v^{\prime}\right) \mathrm{d} v^{\prime}, \\
& \phi(x, v)=0 \quad \text { on } \quad \Gamma_{-}=\{(x, v) \in \partial \Omega \times V, \text { s.t. } v \cdot n(x)<0\}
\end{aligned}
$$

Keywords and phrases: Homogenization, linear transport, eigenvalue problem, drift.

1 Department of Mathematics, University of Chicago, Chicago, IL 60637, U.S.A.; e-mail: gbal@math.uchicago.edu 
posed in a convex bounded open set $\Omega \subset \mathbb{R}^{n}$ and with a compact velocity space $V \subset \mathbb{R}^{n}$. This spectral equation is also called the criticality eigenvalue problem. The vacuum boundary conditions are not very realistic but are assumed here for simplicity. The core is characterized by the absorption coefficient $\Sigma$, the differential scattering coefficient $f$ and the fission coefficient $\sigma$. These coefficients are in practice highly heterogeneous and accurate numerical solutions of (1) still prohibitive even with modern computers. As a first approximation, these coefficients can be assumed periodic. The problem lends then itself naturally to the theory of homogenization, which consists in averaging out the local periodic oscillations and obtaining an equation that only involves the global scale. The numerical solution of this homogenized equation is then significantly less expensive. The homogenization of transport problems has been widely addressed in the past both in the physical $[7,11,16]$ and mathematical $[9,22,23,27,29]$ literature. We $[2,4,5]$ have recently revisited it and obtained new properties of the spectral equation (1).

The homogenization technique proceeds as follows. The density $\phi(x, v)$ is factored as a product of two terms

$$
\phi(x, v)=\psi(x, v) u(x)
$$

Here, $\psi(x, v)$ is a periodic solution of the same spectral transport equation (1) but posed in an infinite medium (or equivalently with periodic boundary conditions at the boundary of the periodicity cell; see (8) below). It characterizes the local oscillations of the density. The function $u(x)$ is the solution of a homogenized diffusion equation posed in the domain $\Omega$. This term gives the global behavior of the density.

In the works mentioned above, the physical coefficients are required to satisfy an additional condition. Let us denote by $\psi^{*}(x, v)$ the solution of the adjoint transport problem to that defining $\psi(x, v)$ (see Eq. (10) below). It is assumed that no intrinsic drift takes place on the periodicity cell $Y$, which is expressed by the relation

$$
J \equiv \int_{Y} \int_{V} v \psi(x, v) \psi^{*}(x, v) \mathrm{d} v \mathrm{~d} x=0 .
$$

This condition is satisfied in most practical applications. For instance, the current $J$ vanishes when the physical parameters satisfy a cubic symmetry in $Y$ and the velocity space verifies $V=-V$. It also vanishes when the cross sections do not depend on the velocity variables $v$ and $v^{\prime}$. We refer to [2] for additional details.

It was shown in [25] however that $J$ does not necessarily vanish when the cell is anisotropic. When (3) is not fulfilled, the particle density is quite different. In [25], where time-dependent problems are considered, the drift is compensated by an exponential increase or decrease of the density in time. A comparable result was formulated in [8] for the heat equation. Here, we would like to compensate for the drift by modifying the amount of fission. In other words, we would like to obtain the fission coefficient and the corresponding criticality constant $k_{\text {eff }}$ that ensures equilibrium. Using an appropriate change of variables, we will show that the eigenvectors of (1) are asymptotically given as the product of a local transport solution and of a large scale solution of a homogeneous diffusion problem with fast exponential decay. Moreover, the criticality constant no longer converges to that of the spectral problem with periodic conditions as in the case with zero drift. Because of an $O(1)$ leak at the boundary of the domain, more fission is required to reach an equilibrium than in the symmetric case and the criticality constant is lowered by the presence of a drift.

Another interest of the present theory is that it devises a homogenization procedure suitable for numerical implementation when the symmetry condition (3) is not satisfied. Most practical calculations of the homogenized diffusion coefficient rely on a compatibility condition of the form (3). We obtain in this paper the derivation of a homogeneous spectral diffusion equation with homogenized diffusion coefficient without assuming any symmetry of the unit cell.

Drift effects have also been analyzed in the homogenization of other equations. In the homogenization of heterogeneous diffusion, parabolic, and transport equations, large drifts are also absent when the physical media satisfy suitable symmetry conditions [8,9]. For more general configurations however, large drift effects may have to be removed first to obtain a limiting homogeneous diffusion equation. Time-dependent and steadystate diffusion equations with drift have been studied in this context $[8,12,13,17,20]$. This paper focuses on the 
criticality spectral equation (1). However, drift effects appear in other linear transport equations. In radiative transfer, the energy density of waves (acoustic, electromagnetic, or seismic waves for instance) propagating in highly heterogeneous media is modeled by a time dependent linear transport equation [28], which after Laplace transform in time has the form

$$
\begin{aligned}
& v \cdot \nabla \phi+\Sigma(x, v) \phi=\int_{V} f\left(x, v^{\prime}, v\right) \phi\left(x, v^{\prime}, \lambda\right) \mathrm{d} v^{\prime}+\lambda \phi \\
& \phi(x, v, \lambda)=0 \quad \text { on } \quad \Gamma_{-}=\{(x, v) \in \partial \Omega \times V, \text { s.t. } v \cdot n(x)<0\}
\end{aligned}
$$

The techniques of this paper can be applied to the analysis of the above spectral equation with asymmetric differential scattering function $f\left(x, v^{\prime}, v\right)$, for which a drift may be induced. See [6] for an application in seismic wave propagation in the absence of drift.

Our framework is the following. We assume that the domain $\Omega$ is composed of the order of $\varepsilon^{-n}$ periodicity cells $\varepsilon Y=(0, \varepsilon)^{n}$, where the unit periodicity cell is $Y=(0,1)^{n}$. Homogenization consists in finding the limit of the particle density and multiplication factor as the number of cells tends to infinity, or equivalently as $\varepsilon$ tends to 0. Physically, the mean free path, the average distance between two interactions of a particle with the underlying medium, is independent of the number of cells in a domain. It is therefore of order $\varepsilon$ in our modeling. This implies that the physical parameters $f, \sigma$, and $\Sigma$, counting a number of interactions by unit length, are of order $\varepsilon^{-1}$. We refer to $[2,15]$ for additional details on this classical scaling. Introducing $\lambda_{\varepsilon}=k_{\text {eff }}^{-1}$, the equation (1) is replaced by the following sequence of criticality problems in $\Omega \times V$ for $\left(\lambda_{\varepsilon}, \phi_{\varepsilon}\right)$

$$
\begin{aligned}
& \varepsilon v \cdot \nabla \phi_{\varepsilon}+\Sigma^{\varepsilon}(x, v) \phi_{\varepsilon}=\int_{V} f^{\varepsilon}\left(x, v^{\prime}, v\right) \phi_{\varepsilon}\left(x, v^{\prime}\right) \mathrm{d} v^{\prime}+\lambda_{\varepsilon} \int_{V} \sigma^{\varepsilon}\left(x, v^{\prime}, v\right) \phi_{\varepsilon}\left(x, v^{\prime}\right) \mathrm{d} v^{\prime}, \\
& \phi_{\varepsilon}=0 \quad \text { on } \quad \Gamma_{-} .
\end{aligned}
$$

Here, the physical parameters are periodic functions in $\Omega$ given by

$$
\Sigma^{\varepsilon}(x, v)=\Sigma\left(\frac{x}{\varepsilon}, v\right), f^{\varepsilon}\left(x, v^{\prime}, v\right)=f\left(\frac{x}{\varepsilon}, v^{\prime}, v\right), \sigma^{\varepsilon}\left(x, v^{\prime}, v\right)=\sigma\left(\frac{x}{\varepsilon}, v^{\prime}, v\right), \quad \text { for } x \in \Omega,
$$

where $\Sigma, f$, and $\sigma$ are positive $Y$-periodic functions of the space variable and are independent of $\varepsilon$. For simplicity, we consider here a vacuum boundary condition for (5). This means that no particles enter the domain at its boundary. Physically more relevant reflection operators at the boundary of the domain that are dissipative (i.e. roughly speaking that enforce that the incoming particle density into the domain is strictly lower than the outgoing density, independent of $\varepsilon$ ) would not modify the analysis carried out here.

An outline of this paper is as follows. Section 1 recalls and extends some results on the spectral equation in finite domain with periodic boundary conditions. Section 2 is devoted to our main results. Using an appropriate change of variables described in Theorem 2.1, we show how to replace (5) by an equivalent spectral equation with zero drift. The classical homogenization theory of [2] then applies, yielding Theorem 2.2. The proof of Theorem 2.1 is based on the properties of exponentially decaying solutions of spectral equations in an infinite medium, which is given in Section 3 .

\section{Existence Results For the SPECTRAL EQUATiOn}

This section states some results of existence and regularity of the solutions of the spectral equation both in finite and periodic domains. Several basic results, such as Theorems 1.1 and 1.2 and Proposition 1.3, have already appeared in the literature $[2-4,15]$. We recall them here for completeness. Theorem 1.4 seems however to be new. This important theorem in the sequel gives an optimal estimate for the smallest eigenvalue of the spectral equation (13) below in terms of the absorption parameter $\Sigma$. Our main hypotheses are as follows. 
(H1) The domain $\Omega$ is a convex bounded open subset in $\mathbb{R}^{n}$.

(H2) The velocity space $V$ is the closure of a bounded open subset in $\mathbb{R}^{n}$ that does not contain 0 with normalized $n$-dimensional measure $|V|=1$.

(H3) Denote by $Y=(0,1)^{n}$ the periodicity cell. The functions $\Sigma(y, v), f\left(y, v^{\prime}, v\right)$, and $\sigma\left(y, v^{\prime}, v\right)$ are $Y$-periodic with respect to the space variable $y$. They are positive bounded functions of their arguments and there exists a positive constant $C>0$ such that, for a.e. $\left(y, v^{\prime}, v\right) \in Y \times V \times V$,

$$
\begin{aligned}
& \sigma\left(y, v^{\prime}, v\right) \geq C, \\
& \Sigma(y, v)-\int_{V} f\left(y, v^{\prime}, v\right) \mathrm{d} v^{\prime} \geq C, \\
& \Sigma(y, v)-\int_{V} f\left(y, v, v^{\prime}\right) \mathrm{d} v^{\prime} \geq C .
\end{aligned}
$$

These assumptions are natural and quite general. They are commented in detail in [2]. We define $W^{p}(\Omega \times V)$, $1 \leq p \leq \infty$, as the following Banach spaces (see [15])

$$
W^{p}(\Omega \times V)=\left\{u \in L^{p}(\Omega \times V) \text { s.t. } v \cdot \nabla u \in L^{p}(\Omega \times V)\right\} \cdot
$$

An existence result for the spectral problem (5) is then the following $[2-4,15]$.

Theorem 1.1. The spectral problem (5) has at most a countable number of eigenvalues and of associated eigenvectors in $W^{2}(\Omega \times V)$. Furthermore, there exists a real and positive eigenvalue, of smallest modulus, with multiplicity one, such that its associated eigenvector is the unique normalized positive eigenvector of (5).

The positive solution of the spectral eigenvalue problem posed in an infinite medium governs the fast scale behavior of the solution in finite domain. We denote by $\lambda_{\infty}$ and $\psi$ the first eigenvalue and eigenvector of the following spectral equations

$$
\begin{aligned}
& T \psi=K \psi+\lambda_{\infty} F \psi \\
& y \mapsto \psi(y, v) \quad \text { is } \quad Y \text {-periodic, }
\end{aligned}
$$

where we have defined

$$
\begin{aligned}
T \phi(y, v) & =v \cdot \nabla_{y} \phi(y, v)+\Sigma(y, v) \phi(y, v) \\
K \phi(y, v) & =\int_{V} f\left(y, v^{\prime}, v\right) \phi\left(y, v^{\prime}\right) \mathrm{d} v^{\prime} \\
F \phi(y, v) & =\int_{V} \sigma\left(y, v^{\prime}, v\right) \phi\left(y, v^{\prime}\right) \mathrm{d} v^{\prime} .
\end{aligned}
$$

Introducing the adjoint functions $f^{*}\left(y, v^{\prime}, v\right)=f\left(y, v, v^{\prime}\right)$ and $\sigma^{*}\left(y, v^{\prime}, v\right)=\sigma\left(y, v, v^{\prime}\right)$, an adjoint problem to (8) is defined as

$$
\begin{aligned}
& T^{*} \psi^{*}=K^{*} \psi^{*}+\lambda_{\infty} F^{*} \psi^{*}, \\
& y \mapsto \psi^{*}(y, v) \text { is } Y-\text { periodic, }
\end{aligned}
$$


where the adjoint operators are defined as

$$
\begin{aligned}
T^{*} \phi(y, v) & =-v \cdot \nabla_{y} \phi(y, v)+\Sigma(y, v) \phi(y, v) \\
K^{*} \phi(y, v) & =\int_{V} f^{*}\left(y, v^{\prime}, v\right) \phi\left(y, v^{\prime}\right) \mathrm{d} v^{\prime} \\
F^{*} \phi(y, v) & =\int_{V} \sigma^{*}\left(y, v^{\prime}, v\right) \phi\left(y, v^{\prime}\right) \mathrm{d} v^{\prime}
\end{aligned}
$$

An existence result for these problems is as follows [2-4].

Theorem 1.2. There exists an eigenvalue $\lambda_{\infty}$ common to both problems (8) and (10), which is real, positive, of smallest modulus, and with multiplicity one. Its associated eigenvectors $\psi$ and $\psi^{*}$ are positive elements of $W^{2}(Y \times V)$. Up to renormalization, $\psi$ and $\psi^{*}$ are the unique positive solutions to (8) and (10), respectively. Furthermore, there exist two positive constants $C^{\prime} \geq C>0$ such that

$$
0<C \leq \psi(y, v) \leq C^{\prime} \quad \text { and } \quad 0<C \leq \psi^{*}(y, v) \leq C^{\prime} \quad \text { a.e. in } Y \times V .
$$

More generally every eigenvector of (8) or (10) in $L^{2}(Y \times V)$ is also an element of $L^{\infty}(Y \times V)$.

We also have the Fredholm alternative:

Proposition 1.3. Let $\lambda_{\infty}$ and $\psi$ be the first eigenvalue and eigenvector of (8). Let $S(y, v)$ be a source term in $L^{2}(Y \times V)$. There exists a solution $\varphi(y, v) \in W^{2}(Y \times V)$ of

$$
\begin{aligned}
& T \varphi=K \varphi+\lambda_{\infty} F \varphi+S \\
& y \mapsto \varphi(y, v) \quad \text { is } \quad Y-\text { periodic }
\end{aligned}
$$

if and only if $S$ is orthogonal to the first eigenvector $\psi^{*}$ of (10), i.e. S satisfies the compatibility condition

$$
\int_{Y} \int_{V} S(y, v) \psi^{*}(y, v) \mathrm{d} y \mathrm{~d} v=0 .
$$

Furthermore, if it exists, the solution $\varphi$ is unique up to the addition of a multiple of $\psi$.

The last two inequalities in (H3) imply that absorption is uniformly greater than scattering; hence multiplication (fission) is necessary to ensure that an equilibrium is reached. This hypothesis on the absorption function is not optimal and we need a more general result. In the analysis of (8), we will need some results on the following similar problem

$$
\begin{aligned}
& T \varphi=\tilde{\mu} \tilde{K} \varphi \\
& y \mapsto \varphi(y, v) \quad \text { is } \quad Y \text {-periodic. }
\end{aligned}
$$

That the analysis of (13) suffices for that of (8) will appear clearly in (49). The operator $\tilde{K}$ is defined as $\tilde{K} \phi(x, v)=\int_{V} \tilde{f}\left(x, v^{\prime}, v\right) \phi\left(x, v^{\prime}\right) \mathrm{d} v^{\prime}$. We seek optimal conditions on the absorption function $\Sigma$ that ensure the well-posedness of this spectral equation and will obtain an estimate for the smallest eigenvalue $\tilde{\mu}$. Let us introduce some notation. For every $(y, v, s) \in Y \times V \times \mathbb{R}$ we define the optical length $\alpha(y, y-s v)$ as

$$
\alpha(y, y-s v)=\int_{0}^{s} \Sigma(y-t v, v) \mathrm{d} t
$$

and the following line integrals

$$
\Pi(\Sigma)(y, v)=\int_{0}^{\infty} \exp (-\alpha(y, y-s v)) \mathrm{d} s
$$


We easily check that $\Pi(\Sigma)$ is $Y$-periodic in the variable $y$. We denote by $L_{\#}^{p}(Y \times V), 1 \leq p \leq \infty$, the spaces of periodic functions in $\mathbb{R}^{n} \times V$ whose restrictions to $Y \times V$ are elements of $L^{p}(Y \times V)$. Hypothesis (H3) is replaced by:

(H3') The functions $\Sigma(y, v)$ and $\tilde{f}\left(y, v^{\prime}, v\right)$ are $Y$-periodic with respect to the space variable $y$. They are bounded functions of their arguments and there exists a positive constant $f_{0}>0$ such that, for a.e. $\left(y, v^{\prime}, v\right) \in$ $Y \times V \times V$,

$$
\tilde{f}\left(y, v^{\prime}, v\right) \geq f_{0} .
$$

(H4) We assume that

$$
\sup _{(y, v) \in Y \times V} \Pi(\Sigma)(y, v)<\infty .
$$

The major advantage of $\left(H 3^{\prime}\right)-(H 4)$ over $(H 3)$ is that $\Sigma$ no longer needs to be positive. It only needs to be positive in some averaged sense.

Theorem 1.4. Assume that (H1), (H2), (H3'), and (H4) hold. Then problem (13) admits a unique positive eigenvector up to renormalization, associated with the smallest eigenvalue $\tilde{\mu}$, which is real and positive. Moreover, we have the following estimate

$$
0<\tilde{\mu} \leq \frac{C}{f_{0}\|\Pi(\Sigma)\|_{L_{\#}^{1}(Y \times V)}^{1 / 2},}
$$

where $C$ is independent of $\Sigma$ and $f_{0}$.

Proof. The functions defined in $Y \times V$ are extended to $\mathbb{R}^{n} \times V$ by $Y$-periodicity. We obtain that

$$
T^{-1}(\phi)=\int_{0}^{\infty} \exp (-\alpha(y, y-s v)) \phi(y-s v, v) \mathrm{d} s .
$$

Therefore $T^{-1}$ is positive and bounded in $L_{\#}^{\infty}(Y \times V)$ since $(\mathrm{H} 4)$ is satisfied. The same results hold for the adjoint operator $T^{*}$ and therefore $T^{-1}$ is bounded in $L_{\#}^{1}(Y \times V)$ and by interpolation [10] in any $L_{\#}^{p}(Y \times V)$ for $1 \leq p \leq \infty$. The operator $\tilde{K}$ is also bounded and positive, and it is a classical result that $T^{-1} \tilde{K}$ is then compact (see $[18,26])$. This is sufficient to obtain the same results as in Theorem 1.2 (see [2-4]).

Let $\varphi$ be the positive solution of $T^{-1} \tilde{K} \varphi=\tilde{\mu}^{-1} \varphi$. We obviously have $\left(T^{-1} \tilde{K}\right)^{2} \varphi=\tilde{\mu}^{-2} \varphi$. By assumption (H2) the interior of $V$ is non-empty. In particular, there exists a velocity $v_{0}$ and a parameter $\delta>0$ such that the following angular sector $\mathcal{S}$ is included in $V$

$$
\mathcal{S}=\left\{v \in \mathbb{R}^{N} \text { s.t. } 1-\delta \leq \frac{|v|}{\left|v_{0}\right|} \leq 1+\delta, \frac{v}{|v|} \cdot \frac{v_{0}}{\left|v_{0}\right|} \geq 1-\delta\right\} \subset V .
$$

For $y \in \mathbb{R}^{n}$, we define the infinite cone of origin $y$ as $\left\{y+s v\right.$ s.t. $\left.s \in \mathbb{R}^{+}, v \in \mathcal{S}\right\}$. By virtue of hypotheses (H2) and (H3'), we denote by $\Sigma_{\max }$ and $v_{\max }$ the maximum of $|\Sigma(x, v)|$ on $\Omega \times V$ and $|v|$ on $V$, respectively. We then obtain that

$$
\begin{aligned}
\tilde{K} T^{-1} \tilde{K}(\varphi)(y) & \geq f_{0}^{2} \int_{V} \mathrm{~d} v \int_{0}^{\infty} \mathrm{d} s \exp (-\alpha(y, y-s v)) \int_{V} \mathrm{~d} v^{\prime} \varphi\left(y-s v, v^{\prime}\right) \\
& \geq C \int_{C_{\mathcal{S}}} \mathrm{d} z \frac{\exp \left(-\frac{\Sigma_{\max }}{v_{\max }}|z-y|\right)}{|z-y|^{n-1}} \int_{V} \mathrm{~d} v^{\prime} \varphi\left(z, v^{\prime}\right),
\end{aligned}
$$


owing to the change of variables $z=y-s v$ from polar to Cartesian coordinates. Clearly the cone $C_{\mathcal{S}}$ contains at least one periodicity cell $Y$ and therefore

$$
\tilde{K} T^{-1} \tilde{K}(\varphi)(y) \geq C \int_{Y} \int_{V} \varphi\left(z, v^{\prime}\right) \mathrm{d} v^{\prime} \mathrm{d} z=C\|\varphi\|_{L^{1}(Y \times V)},
$$

since $\varphi$ is positive. Finally we obtain that

$$
\begin{aligned}
\tilde{\mu}^{-2}\|\varphi\|_{L^{1}(Y \times V)} & =\left\|T^{-1} \tilde{K} T^{-1} \tilde{K}(\varphi)\right\|_{L^{1}(Y \times V)} \geq C\|\varphi\|_{L^{1}(Y \times V)}\left\|T^{-1}(1)\right\|_{L^{1}(Y \times V)} \\
& \geq C\|\varphi\|_{L^{1}(Y \times V)}\|\Pi(\Sigma)\|_{L^{1}(Y \times V)} .
\end{aligned}
$$

This concludes the proof of the estimate.

The estimate for $\tilde{\mu}$ given in the theorem may not be optimal (the exponent $1 / 2$ may not be optimal). However, we obtain that $\tilde{\mu}$ tends to 0 as $\Pi(\Sigma)(y, v)$ goes to infinity on a fixed set of positive measure. This proves that hypothesis (H4) is optimal in this sense since the smallest eigenvalue of (13) cannot be positive when (H4) is violated.

\section{Homogenization of THE SPECTRAL EQUATION With DRIFT}

This section states our main results in Theorems 2.1 and 2.2 below. We begin with a formal derivation of the diffusion equation that yields the correct result in the absence of drift, that is to say when the physical parameters satisfy enough symmetry constraints. It is based on the classical two-scale expansion, which has often been used to obtain the asymptotic limit of heterogeneous transport equations with periodic coefficients $[2,4,7,9,15,22-24]$.

The homogenization of the transport equation without drift proceeds as follows. We define

$$
\psi_{\varepsilon}(x, v)=\psi\left(\frac{x}{\varepsilon}, v\right)
$$

where $\psi$ is the positive solution of the local spectral equation (8). Since the medium is periodic, we can expect that the solutions of (5) and (8) share the same fast-scale oscillations. Since $\psi_{\varepsilon}$ is a positive function, we introduce the factored density $u_{\varepsilon}$

$$
u_{\varepsilon}(x, v)=\frac{\phi_{\varepsilon}(x, v)}{\psi_{\varepsilon}(x, v)}
$$

where $\phi_{\varepsilon}$ is an eigenvector of (5). After some algebra, the equation for $u_{\varepsilon}$ is found to be

$$
\begin{aligned}
& \frac{1}{\varepsilon} v \cdot \nabla u_{\varepsilon}+\frac{1}{\varepsilon^{2}} \int_{V} \sigma_{\infty}^{\varepsilon}\left(x, v^{\prime}, v\right) \frac{\psi_{\varepsilon}\left(x, v^{\prime}\right)}{\psi_{\varepsilon}(x, v)}\left[u_{\varepsilon}(x, v)-u_{\varepsilon}\left(x, v^{\prime}\right)\right] \mathrm{d} v^{\prime} \\
= & \frac{\lambda_{\varepsilon}-\lambda_{\infty}}{\varepsilon^{2}} \int_{V} \sigma^{\varepsilon}\left(x, v^{\prime}, v\right) \frac{\psi_{\varepsilon}\left(x, v^{\prime}\right)}{\psi_{\varepsilon}(x, v)} u_{\varepsilon}\left(x, v^{\prime}\right) \mathrm{d} v^{\prime}
\end{aligned}
$$

where $\sigma_{\infty}^{\varepsilon}=f^{\varepsilon}+\lambda_{\infty} \sigma^{\varepsilon}$. The two-scale method consists then in assuming the following expansion,

$$
\begin{aligned}
u_{\varepsilon}(x, v) & =u_{0}\left(x, \frac{x}{\varepsilon}, v\right)+\varepsilon u_{1}\left(x, \frac{x}{\varepsilon}, v\right)+\varepsilon^{2} u_{2}\left(x, \frac{x}{\varepsilon}, v\right)+\ldots \\
\lambda_{\varepsilon} & =\lambda_{0}+\varepsilon \lambda_{1}+\varepsilon^{2} \lambda_{2}+\ldots,
\end{aligned}
$$


where the functions $y \mapsto u_{k}(x, y, v)$ are $Y$-periodic for $k=0, \ldots, 2$. Here, the variable $x$ corresponds to the global scale whereas $y=\frac{x}{\varepsilon}$ describes the local fluctuations of the particle density. Plugging this expansion (19) into the transport equation (18) and equating like powers of $\varepsilon$ yields the following three equations

$$
\begin{aligned}
& v \cdot \nabla_{y} u_{0}+Q\left(u_{0}\right)=\left(\lambda_{0}-\lambda_{\infty}\right) \tilde{F}\left(u_{0}\right) \\
& v \cdot \nabla_{y} u_{1}+Q\left(u_{1}\right)=-v \cdot \nabla_{x} u_{0}+\lambda_{1} \tilde{F}\left(u_{0}\right) \\
& v \cdot \nabla_{y} u_{2}+Q\left(u_{2}\right)=-v \cdot \nabla_{x} u_{1}+\lambda_{1} \tilde{F}\left(u_{1}\right)+\lambda_{2} \tilde{F}\left(u_{0}\right) .
\end{aligned}
$$

The scattering operator $Q$ and fission operator $\tilde{F}$ are defined as

$$
\begin{aligned}
& Q(u)(y, v)=\int_{V} \sigma_{\infty}\left(y, v^{\prime}, v\right) \frac{\psi\left(y, v^{\prime}\right)}{\psi(y, v)}\left[u(y, v)-u\left(y, v^{\prime}\right)\right] \mathrm{d} v^{\prime} \\
& \tilde{F}(u)(y, v)=\int_{V} \sigma\left(y, v^{\prime}, v\right) \frac{\psi\left(y, v^{\prime}\right)}{\psi(y, v)} u\left(y, v^{\prime}\right) \mathrm{d} v^{\prime},
\end{aligned}
$$

where $\sigma_{\infty}=f+\lambda_{\infty} \sigma$. We now want to solve the three equations $(20,21)$, and (22) in turn. When one of these equations does not admit any solution, this is an indication that the expansion (19) does not hold or becomes trivial. This is precisely what will happen when a drift is present.

We first deduce from (20), Theorem 1.2, and Proposition 1.3 that $\lambda_{0}=\lambda_{\infty}$ and $u_{0} \equiv u_{0}(x)$. As expected, the fast oscillations of $\phi_{\varepsilon}$ are captured by the local eigenfunction $\psi_{\varepsilon}$ and $u_{\varepsilon}$ converges (strongly) to a function that does not depend on the fast scale. We obtain from the second equation (21) and the same Fredholm alternative in Proposition 1.3 that

$$
\int_{Y} \int_{V}\left(-v \cdot \nabla_{x} u_{0}+\lambda_{1} \tilde{F}\left(u_{0}\right)\right) \psi(y, v) \psi^{*}(y, v) \mathrm{d} y \mathrm{~d} v=0 .
$$

Since $u_{0}$ in independent of $y$ and $v$, the latter is equivalent to

$$
J \cdot \nabla u_{0}=\lambda_{1} \tilde{\sigma} u_{0}
$$

where $\tilde{\sigma}=\int_{Y} \int_{V} \tilde{F}(1) \psi(y, v) \psi^{*}(y, v) \mathrm{d} y \mathrm{~d} v$ is a positive constant and

$$
J=\int_{Y} \int_{V} v \psi(y, v) \psi^{*}(y, v) \mathrm{d} y \mathrm{~d} v .
$$

When $J=0$, we obtain that $\lambda_{1}=0$. Then solving (21) for $u_{1}$ and plugging its expression into (22) gives a compatibility condition which is a spectral diffusion equation for $u_{0}$. This equation is similar to (29) below. Although only formal here, this procedure can be justified rigorously $[4,15]$ provided the domain is sufficiently regular. The regularity constraint can be circumvented by using the method of two-scale convergence $[1,2]$. The final result can be read off from Theorem 2.2 by setting $\nu=0$.

When $J \neq 0$, we obtain that $u_{0}$ is exponentially increasing or decreasing in the direction $J$. However the boundary conditions impose that $u_{0}=0$ at the boundary. Therefore $u_{0} \equiv 0$ and the asymptotic expansion fails to give the right answer. It is proved in [2] that the solution $u_{\varepsilon}$ of an associated source problem indeed converges strongly to 0 when $J \neq 0$.

We deduce from the previous asymptotic expansion that $\psi(y, v)$ does not reproduce the local behavior of the solution $\phi_{\varepsilon}$ since the expansion for $u_{\varepsilon}$ yields a trivial result. Also, the expression in (24) shows that the leading term "wants" to decay exponentially. These observations lead us to the following modifications. We want to impose an exponential decay of the solution by introducing the following change of variables

$$
\varphi_{\varepsilon, \nu}=\exp \left(\frac{\nu \cdot x}{\varepsilon}\right) \phi_{\varepsilon}
$$


where $\nu$ is a vector in $\mathbb{R}^{n}$. We readily verify that the equation for $\varphi_{\varepsilon, \nu}$ is still (5), where $\Sigma^{\varepsilon}$ has been replaced by $\Sigma^{\varepsilon}-v \cdot \nu$. The objective is to find a vector $\nu$ that allows us to use the known results for vanishing drift. Let us introduce $\lambda_{\infty, \nu}, \psi_{\nu}$, and $\psi_{\nu}^{*}$, the smallest eigenvalue and corresponding positive eigenvectors of the two following spectral equations

$$
\begin{aligned}
& T \psi_{\nu}-v \cdot \nu \psi_{\nu}=K \psi_{\nu}+\lambda_{\infty, \nu} F \psi_{\nu} \\
& y \mapsto \psi_{\nu}(y, v) \quad \text { is } \quad Y \text {-periodic }
\end{aligned}
$$

and

$$
\begin{aligned}
& T^{*} \psi_{\nu}^{*}-v \cdot \nu \psi_{\nu}^{*}=K^{*} \psi_{\nu}^{*}+\lambda_{\infty, \nu} F^{*} \psi_{\nu}^{*} \\
& y \mapsto \psi_{\nu}^{*}(y, v) \quad \text { is } \quad Y \text { - periodic. }
\end{aligned}
$$

We want to find $\nu$ such that

$$
J_{\nu}=\int_{Y} \int_{V} v \psi_{\nu}(y, v) \psi_{\nu}^{*}(y, v) \mathrm{d} y \mathrm{~d} v=0
$$

Notice that similar techniques have been used for parabolic problems $[8,21]$ or in the homogenization of heterogeneous elliptic spectral equations $[12,13]$. That such a vector $\nu$ exists is stated in our main result:

Theorem 2.1. There exists a unique vector $\nu \in \mathbb{R}^{n}$ such that the spectral equations (26) and (27) are well-posed (in the sense that the conclusions of Th. 1.2 apply), such that (28) holds, and such that $\lambda_{\infty, \nu} \geq 0$. Moreover, if $J \neq 0$, the leading eigenvalue $\lambda_{\infty, \nu}$ satisfies

$$
\lambda_{\infty, \nu}>\lambda_{\infty}=\lambda_{\infty, 0} .
$$

This theorem is proved in Section 3. Thanks to this theorem, we have the right change of variables (25) that allows us to replace a problem with large drift by a problem with vanishing drift. We can then apply known results on the homogenization of the spectral transport equation without drift. Let us define the $\varepsilon Y$-periodic function $\psi_{\varepsilon, \nu}$ as

$$
\psi_{\varepsilon, \nu}(x, v)=\psi_{\nu}\left(\frac{x}{\varepsilon}, v\right),
$$

where $\psi_{\nu}$ is the positive eigenvector of (26). In our context, Theorem 3.1 in [2] now reads:

Theorem 2.2. Let the vector $\nu$ be given as in Theorem 2.1. Let $\lambda_{\varepsilon}^{k}$ be the $k^{\text {th }}$ eigenvalue of (5) and let $\phi_{\varepsilon}^{k}$ be a normalized associated eigenvector. Then

$$
\lim _{\varepsilon \rightarrow 0} \frac{\lambda_{\varepsilon}^{k}-\lambda_{\infty, \nu}}{\varepsilon^{2}}=\rho^{k}
$$

where $\lambda_{\infty, \nu}$ is the first eigenvalue of (26) and $\rho^{k}$ is the $k^{\text {th }}$ eigenvalue of the homogenized diffusion problem

$$
\begin{array}{rll}
-\nabla \cdot D \nabla u^{k}(x) & =\rho^{k} \bar{\sigma} u^{k}(x) & \text { in } \Omega \\
u^{k}(x) & =0 & \text { on } \partial \Omega .
\end{array}
$$

Furthermore, up to a subsequence,

$$
\frac{\phi_{\varepsilon}^{k}(x, v) \exp \left(\frac{\nu \cdot x}{\varepsilon}\right)}{\psi_{\varepsilon, \nu}(x, v)} \rightarrow u^{k}(x) \text { strongly in } L^{2}(\Omega \times V),
$$


where $u^{k}$ is an eigenvector associated with the eigenvalue $\rho^{k}$. The homogenized fission cross section is given by

$$
\bar{\sigma}=\int_{Y} \int_{V} \int_{V} \sigma\left(y, v^{\prime}, v\right) \psi_{\nu}^{*}\left(y, v^{\prime}\right) \psi_{\nu}(y, v) \mathrm{d} y \mathrm{~d} v \mathrm{~d} v^{\prime}
$$

while the homogenized diffusion tensor $D=\left(D_{i j}\right)_{1 \leq i, j \leq N}$ in (29) is defined by

$$
D_{i j}=-\int_{Y} \int_{V} v_{j} \psi_{\nu}(y, v) \psi_{\nu}^{*}(y, v) \theta_{\nu}^{i}(y, v) \mathrm{d} v \mathrm{~d} y
$$

and the functions $\left(\theta_{\nu}^{i}\right)_{1 \leq i \leq N}$ are the solutions of the cell problems

$$
\begin{aligned}
& v \cdot \nabla_{y} \theta_{\nu}^{i}+Q_{\nu}\left(\theta_{\nu}^{i}\right)=-v_{i} \quad \text { in } Y \times V \\
& y \mapsto \theta_{\nu}^{i}(y, v) \text { is } Y \text {-periodic, }
\end{aligned}
$$

where the local scattering operator $Q_{\nu}$ is defined by

$$
Q_{\nu}(\theta)=\int_{V} \frac{\psi_{\nu}\left(y, v^{\prime}\right)}{\psi_{\nu}(y, v)} \sigma_{\infty, \nu}\left(y, v^{\prime}, v\right)\left[\theta(y, v)-\theta\left(y, v^{\prime}\right)\right] \mathrm{d} v^{\prime}
$$

with the notation $\sigma_{\infty, \nu}\left(y, v^{\prime}, v\right)=f\left(y, v^{\prime}, v\right)+\lambda_{\infty, \nu} \sigma\left(y, v^{\prime}, v\right)$.

The labeling of the eigenvalues of (5) are made by increasing order of their real part. These eigenvalues may be complex; however in the limit of small mean free path, they are all real. The eigenvalues of the diffusion equation (29) are real and labeled by increasing order.

We can recover the separation of scales (2) given in the introduction. Indeed, as a corollary of the previous theorem, we easily show that

$$
\phi_{\varepsilon}^{k}(x, v)=\psi_{\varepsilon, \nu}(x, v) u_{\varepsilon}^{k}(x)+\text { lower order terms }
$$

where $u_{\varepsilon}^{k}(x)=\exp \left(-\varepsilon^{-1} \nu \cdot x\right) u^{k}(x)$ is a solution of the following convection-diffusion spectral equation

$$
\begin{aligned}
-\nabla \cdot D \nabla u_{\varepsilon}^{k}(x)-\frac{2}{\varepsilon} \nu \cdot D \nabla u_{\varepsilon}^{k}(x)-\frac{\nu \cdot D \nu}{\varepsilon^{2}} u_{\varepsilon}^{k}(x) & =\rho^{k} \bar{\sigma} u_{\varepsilon}^{k}(x) \quad \text { in } \Omega \\
u_{\varepsilon}^{k}(x) & =0 \quad \text { on } \partial \Omega .
\end{aligned}
$$

Therefore in a certain sense, there is still separation of scales. The solution of the transport equation is approximated by the product of a fast oscillating function and of a smooth function, which now decays exponentially in the fast scale. Notice that the local behavior of the particle density is governed by $\psi_{\varepsilon, \nu}(x, v)$, and no longer by $\psi_{\varepsilon, 0}(x, v)$ as in the case of vanishing drift. Therefore, the expansion for $u_{\varepsilon}$ defined in (18) cannot lead to the right answer.

Let us also give a qualitative remark concerning the density at the boundary of the domain. Notice that the outgoing density at the boundary of the domain $\Omega$ has not been estimated. The Dirichlet condition for the convection-diffusion approximation and the relation (33) yield a vanishing exiting flux which is not physical. The size of the exiting flux is known in the case of a similar source problem [4] with vanishing drift. In this case, the exiting flux is shown to be of order $\varepsilon$ when the $L^{2}$ norm of the solution of the transport equation is of order 1. Up to the addition of a boundary layer that we do not consider here, the transport solution is approximated by a diffusion term $w_{\varepsilon}$ satisfying an equation in $\Omega$ similar to (29) with Robin boundary condition

$$
w_{\varepsilon}+\varepsilon L \frac{\partial w_{\varepsilon}}{\partial n}=0
$$


where the extrapolation length $L$ is positive. Hence, the diffusion term is of order $\varepsilon$ at the boundary of the domain. The solution of the transport equation is given by

$$
\phi_{\varepsilon}(x, v)=\psi_{\varepsilon, \nu}(x, v) \exp \left(\frac{\nu \cdot x}{\varepsilon}\right) w_{\varepsilon}(x)+\text { boundary layer }+ \text { lower order terms. }
$$

Assume that $\Omega=(0,1)^{n}$ and to simplify that the components of $\nu$ are non-positive, $\nu_{i}<0$ for $1 \leq i \leq n$. Then the drift directs the particles towards the sides of $\Omega$ where $x_{i}=0$. In order to have a normalized solution $\phi_{\varepsilon}$ of norm 1 in $L^{2}(\Omega \times V)$, the $L^{2}$ norm of $w_{\varepsilon}$ must be $O\left(\varepsilon^{-1}\right)$. Therefore we obtain that the exiting distribution of particles $\phi_{\varepsilon}(x, v)$ is of order 1 on the sides $x_{i}=0$ instead of being of order $\varepsilon$ when the drift vanishes.

As a consequence, the condition of vanishing incoming flux of particles implies that a boundary layer of $O(1)$ is unavoidable. Indeed, the transport solution is very anisotropic at the vicinity of the boundary. It is of order one on the sides $x_{i}=0$ for the exiting directions, and vanishing for the incoming directions. Therefore the computation of the boundary layer derived in [4] is necessary to accurately describe the exiting density. Notice also that the leakage of particles at the boundary of the domain is of $O(1)$ because the exiting flux is of $O(1)$. This is consistent with $\lambda_{\infty, \nu}>\lambda_{\infty}$. More fission is needed to compensate for leakage. The criticality constant $k_{\text {eff }}$ in (1) is therefore lowered when some drift is generated by the anisotropy of the physical coefficients.

\section{Exponentially DECAYING SOLUTIONS IN INFINITE MEDIUM}

This section is devoted to the proof of Theorem 2.1. Our objective is to derive exponentially decaying solutions of the spectral equation in the infinite periodic domain for which the drift term (28) vanishes. The parameter at our disposal is the vector $\nu$ pointing in the direction in which the solution decays. We are therefore seeking the smallest eigenvalue and the associated positive eigenvector of the following sequence of problems parameterized by $\nu$ :

$$
\begin{aligned}
& T \phi_{\nu}=K \phi_{\nu}+\lambda_{\infty, \nu} F \phi_{\nu} \\
& y \mapsto \exp (\nu \cdot y) \phi_{\nu}(y, v) \quad \text { is } \quad Y \text { - periodic. }
\end{aligned}
$$

Notice that this family of periodic problems is similar to the one defined in [8] (Sect. 4.5.8), where the heat equation is analyzed. Unlike the results of this reference, we have not been able to show that the function $\nu \mapsto-\lambda_{\infty, \nu}$ is strictly convex (and this may actually not be true). This is consistent with the results in [13]. However, this convexity property is not necessary to ensure uniqueness of the vector $\nu$ such that the eigenvalue $\lambda_{\infty, \nu}$ is positive and the drift term (28) vanishes. Introducing the change of variables $\psi_{\nu}(y, v)=\exp (\nu \cdot y) \phi_{\nu}(y, v)$, we recast (35) as

$$
\begin{aligned}
& T \psi_{\nu}-v \cdot \nu \psi_{\nu}=K \psi_{\nu}+\lambda_{\infty, \nu} \psi_{\nu} \\
& y \mapsto \psi_{\nu}(y, v) \quad \text { is } \quad Y-\text { periodic. }
\end{aligned}
$$

Before analyzing (36), we consider an auxiliary simpler problem where scattering is absent. Introduce the following sequence of eigenvalue problems

$$
\begin{aligned}
& T \varphi_{\nu}-v \cdot \nu \varphi_{\nu}=\tilde{\mu}_{\nu} \tilde{K} \varphi_{\nu} \\
& y \mapsto \varphi_{\nu}(y, v) \quad \text { is } \quad Y \text { - periodic. }
\end{aligned}
$$

The usual sequence of adjoint eigenvalue problems is given by

$$
\begin{aligned}
& T^{*} \varphi_{\nu}^{*}-v \cdot \nu \varphi_{\nu}^{*}=\tilde{\mu}_{\nu} \tilde{K}^{*} \varphi_{\nu}^{*} \\
& y \mapsto \varphi_{\nu}^{*}(y, v) \quad \text { is } \quad Y-\text { periodic. }
\end{aligned}
$$


The set of admissible values of $\nu$ is such that the absorption $\Sigma-v \cdot \nu$ remains positive on average (in the sense of $(H 4))$ since we are interested only in positive values of $\tilde{\mu}_{\nu}$. We consequently define the set

$$
\Lambda=\left\{\nu \in \mathbb{R}^{n} \text {, s.t. }(\Sigma-v \cdot \nu)(y, v) \text { satisfies }(\mathrm{H} 4)\right\} \text {. }
$$

We now state the main result on the function $\tilde{\mu}_{\nu}$.

Proposition 3.1. Assume that hypotheses (H1), (H2), and (H3') hold. We define the function $\nu \mapsto \tilde{\mu}_{\nu}$ with domain of definition $\Lambda$ as the smallest eigenvalue of (37). We denote by $\varphi_{\nu}$ and $\varphi_{\nu}^{*}$ the corresponding positive normalized eigenvectors of (37) and (38), respectively.

Then, we have that $\nu \mapsto \tilde{\mu}_{\nu}$ is twice continuously differentiable and attains its maximal value at a unique point $\nu_{0}$. We further have that

$$
\frac{\partial \tilde{\mu}_{\nu}}{\partial \nu}\left(\nu_{0}\right)=0 \quad \text { and } \quad-\frac{\partial^{2} \tilde{\mu}_{\nu}}{\partial \nu_{i} \partial \nu_{j}}\left(\nu_{0}\right) \quad \text { is a positive definite } n \times n \text { matrix. }
$$

Moreover, the drift term vanishes

$$
\tilde{J}_{\nu_{0}}=\int_{Y} \int_{V} v \varphi_{\nu_{0}}(y, v) \varphi_{\nu_{0}}^{*}(y, v) \mathrm{d} y \mathrm{~d} v=0 .
$$

Proof. The first step is to show some regularity properties of the function $\nu \mapsto\left(\tilde{\mu}_{\nu}, \varphi_{\nu}\right)$. The general theory of $[14,19]$ can be utilized to obtain most of the results of regularity presented here. Since the proof of local convexity requires the same calculations, we present them in detail now. Notice that analogous results have been obtained in $[8]$ in the framework of second-order elliptic equations.

We first deduce from Theorem 1.4 the existence of $\left(\tilde{\mu}_{\nu}, \varphi_{\nu}\right)$ for $\nu \in \Lambda$. Let now $\nu \in \Lambda$ and $\delta \nu \in \mathbb{R}^{n}$ such that $\nu+\delta \nu \in \Lambda$. For a sequence of functions $\theta_{\nu}$, we define $\delta \theta_{\nu}=\theta_{\nu+\delta \nu}-\theta_{\nu}$. We deduce from (37) that

$$
\left(T-v \cdot \nu-\tilde{\mu}_{\nu} \tilde{K}\right) \delta \varphi_{\nu}=v \cdot \delta \nu \varphi_{\nu+\delta \nu}+\delta \tilde{\mu}_{\nu} \tilde{K} \varphi_{\nu+\delta \nu} .
$$

From the Fredholm alternative of Proposition 1.3, we have

$$
\delta \tilde{\mu}_{\nu}\left(\tilde{K} \varphi_{\nu+\delta \nu}, \varphi_{\nu}^{*}\right)=-\delta \nu \cdot\left(v \varphi_{\nu+\delta \nu}, \varphi_{\nu}^{*}\right) .
$$

Here $(\cdot, \cdot)$ is the usual scalar product in $L^{2}(Y \times V)$. Since both $\varphi_{\nu+\delta \nu}$ and $\varphi_{\nu}^{*}$ are positive functions, we deduce that

$$
\left|\tilde{\mu}_{\nu+\delta \nu}-\tilde{\mu}_{\nu}\right| \leq C|\delta \nu|,
$$

where $C$ is independent of $\delta \nu$; hence the continuity of $\nu \mapsto \tilde{\mu}_{\nu}$. We then deduce from equation (40) and Proposition 1.3 that

$$
\delta \varphi_{\nu}=C \varphi_{\nu}+O(\delta \nu) .
$$

Since $\varphi_{\nu}$ and $\varphi_{\nu+\delta \nu}$ are normalized, we deduce that $C=O(\delta \nu)$ and the continuity of $\nu \mapsto \varphi_{\nu}$. Again owing to $(41,40)$, and the continuity of $\nu \mapsto\left(\tilde{\mu}_{\nu}, \varphi_{\nu}\right)$, we obtain that this map also differentiable. The equation for $\frac{\partial}{\partial \nu} \varphi_{\nu}$ is defined up to a constant. Upon differentiating the normalization equation $\int_{Y} \int_{V}\left(\varphi_{\nu}\right)^{2} \mathrm{~d} y \mathrm{~d} v=1$, we easily deduce that this constant vanishes. We thus obtain that

$$
\nabla_{\nu} \tilde{\mu}_{\nu}=-\bar{\sigma}_{\nu}^{-1} \tilde{J}_{\nu}
$$


where $\bar{\sigma}_{\nu}=\int_{Y} \int_{V} K\left(\varphi_{\nu}\right) \varphi_{\nu}^{*} \mathrm{~d} y \mathrm{~d} v>0$ and the drift current $\tilde{J}_{\nu}$ is defined as

$$
\tilde{J}_{\nu}=\int_{Y} \int_{V} v \varphi_{\nu}(y, v) \varphi_{\nu}^{*}(y, v) \mathrm{d} y \mathrm{~d} v
$$

We define

$$
\varphi_{\nu, \nu_{i}}=\frac{\partial}{\partial \nu_{i}} \varphi_{\nu} \quad \text { and } \quad \tilde{\mu}_{\nu, \nu_{i}}=\frac{\partial}{\partial \nu_{i}} \tilde{\mu}_{\nu}
$$

We deduce from (40) that

$$
\left(T-v \cdot \nu-\tilde{\mu}_{\nu} \tilde{K}\right) \nabla_{\nu} \varphi_{\nu}=v \varphi_{\nu}+\nabla_{\nu} \tilde{\mu}_{\nu} \tilde{K} \varphi_{\nu}
$$

Therefore, we have

$$
\left(T-v \cdot \nu-\tilde{\mu}_{\nu} \tilde{K}\right)\left(\delta \nabla_{\nu} \varphi_{\nu}\right)=v \cdot \delta \nu \varphi_{\nu+\delta \nu, \nu_{i}}+v_{i} \delta \varphi_{\nu}+\tilde{K}\left(\varphi_{\nu+\delta \nu, \nu_{i}} \delta \tilde{\mu}_{\nu}+\tilde{\mu}_{\nu, \nu_{i}} \delta \varphi_{\nu}+\varphi_{\nu} \delta \tilde{\mu}_{\nu, \nu_{i}}\right) .
$$

Using the same Fredholm alternative as above, we deduce that $\delta \tilde{\mu}_{\nu, \nu_{i}}$, and then $\delta \phi_{\nu, \nu_{i}}$ are of order $O(d \nu)$. Therefore, $\tilde{\mu}_{\nu}$ is twice differentiable and

$$
\bar{\sigma}_{\nu} \frac{\partial^{2}}{\partial \nu_{j} \partial \nu_{i}} \tilde{\mu}_{\nu}=-\left(v_{i} \zeta \varphi_{\nu, \nu_{j}}+v_{j} \varphi_{\nu, \nu_{i}}+\tilde{K}\left(\tilde{\mu}_{\nu, \nu_{j}} \varphi_{\nu, \nu_{i}}+\tilde{\mu}_{\nu, \nu_{i}} \varphi_{\nu, \nu_{j}}\right), \varphi_{\nu}^{*}\right) .
$$

Let us now define

$$
D_{i j}(\nu)=-\frac{\partial^{2} \tilde{\mu}_{\nu}}{\partial \nu_{i} \partial \nu_{j}}
$$

This tensor is clearly symmetric. Iterating the above technique one more time, we also show that it depends continuously on $\nu \in \Lambda$.

Since $0 \in \Lambda$ and $\tilde{\mu}_{0}>0$, and since $\tilde{\mu}_{\nu}$ tends to 0 as $\nu$ approaches the boundary of $\Lambda$ thanks to Theorem 1.4, we deduce that the continuous function $\nu \rightarrow \tilde{\mu}_{\nu}$ reaches its maximum in the interior of $\Lambda$, say at $\nu_{0}$. We do not have uniqueness of the point $\nu_{0}$ yet. However, we have that $\tilde{\mu}_{\nu_{0}, \xi}=0$. Let us show that this implies the positive definiteness of $D_{i j}\left(\nu_{0}\right)$. Upon multiplying (42) by $\varphi_{\nu, \xi} \varphi_{\nu}^{*}\left(\varphi_{\nu}\right)^{-1}$ at $\nu=\nu_{0}$, we deduce from (44) and (45) that

$$
\bar{\sigma}_{\nu_{0}} D\left(\nu_{0}\right) \xi \cdot \xi=2 \int_{Y \times V}\left(T \varphi_{\nu_{0}, \xi}-v \cdot \nu_{0} \varphi_{\nu_{0}, \xi}-\tilde{\mu}_{\nu_{0}} \tilde{K} \varphi_{\nu_{0}, \xi}\right) \frac{\varphi_{\nu_{0}, \xi}}{\varphi_{\nu_{0}}} \varphi_{\nu_{0}}^{*} \mathrm{~d} y \mathrm{~d} v
$$

Notice that this equality holds at every point $\nu$ such that $\tilde{\mu}_{\nu, \xi}=0$. Keeping this in mind, we drop the index 0 and define $\theta=\frac{\varphi_{\nu, \xi}}{\varphi_{\nu}}$. Since $\varphi_{\nu}$ is a positive solution of (37), we obtain that

$$
\bar{\sigma}_{\nu} D(\nu) \xi \cdot \xi=2 \int_{Y \times V}\left(v \cdot \nabla_{y} \theta+\tilde{\mu}_{\nu}\left[\frac{\theta}{\varphi_{\nu}} \tilde{K}\left(\varphi_{\nu}\right)-\frac{1}{\varphi_{\nu}} \tilde{K}\left(\varphi_{\nu} \theta\right)\right]\right) \theta \varphi_{\nu} \varphi_{\nu}^{*} \mathrm{~d} y \mathrm{~d} v
$$

We deduce from (37) and its adjoint equation that

$$
v \cdot \nabla\left(\varphi_{\nu} \varphi_{\nu}^{*}\right)=\tilde{\mu}_{\nu}\left(\varphi_{\nu}^{*} \tilde{K}\left(\varphi_{\nu}\right)-\varphi_{\nu} \tilde{K}^{*}\left(\varphi_{\nu}^{*}\right)\right) .
$$

Integrating (47) by parts using the above relation yields after some algebra that

$$
\bar{\sigma}_{\nu} D(\nu) \xi \cdot \xi=\int_{Y \times V} \int_{Y} f\left(y, v^{\prime}, v\right) \varphi_{\nu}^{*}(y, v) \varphi_{\nu}\left(y, v^{\prime}\right)\left|\theta\left(y, v^{\prime}\right)-\theta(y, v)\right|^{2} \mathrm{~d} v^{\prime} \mathrm{d} y \mathrm{~d} v .
$$


The same techniques are used in $[2,4]$ to obtain crucial a priori estimates that are used to deal with the spectral transport equation in the absence of drift. Therefore, $D(\nu)$ is positive. Assume now that $D \xi \cdot \xi=0$. Then from (48), $\theta$ is independent of $v$. We deduce then from (42) that

$$
v \cdot \nabla_{y} \theta=v \cdot \xi
$$

Upon integrating this equality over the cell $Y$, we get that $\xi \cdot v=0$ for every $v \in V$, which, owing to hypothesis $(\mathrm{H} 2)$, yields $\xi=0$. Therefore, $D(\nu)$ is positive definite at every point $\nu$ such that $\tilde{\mu}_{\nu, \xi}=0$. By continuity, we obtain the local convexity of the function $\nu \rightarrow-\tilde{\mu}_{\nu}$ in the vicinity of such points. This actually implies the uniqueness of the point $\nu_{0}$ such that $\tilde{\mu}_{\nu, \xi}=0$. Indeed, the domain $\Lambda$ is clearly connected and star-like by construction. Assume now that $\nu \rightarrow \tilde{\mu}_{\nu}$ has two local maxima. Since $\tilde{\mu}_{\nu}$ is a $C^{2}$ function that vanishes at the boundary of $\Lambda$, there exists at least one point where its gradient vanishes and its Hessian matrix cannot be negative definite (for the existence of a saddle point, see [30], Chap. II, Th. I.1). However, $\tilde{\mu}_{\nu_{m}, \xi}=0$ and then $D\left(\nu_{m}\right)$ is positive definite, which is a contradiction. We deduce that there exists a unique point $\nu_{0}$ such that $\tilde{\mu}_{\nu_{0}, \xi}=0$ and equivalently such that $\tilde{J}_{\nu_{0}}=0$. This concludes the proof of this proposition.

We are now in a position to prove Theorem 2.1. To use the results of the above proposition, we introduce the following two-parameter family of eigenvalue problems

$$
\begin{aligned}
& T \psi(\lambda, \nu)-v \cdot \nu \psi(\lambda, \nu)=\mu(\lambda, \nu)(K+\lambda F) \psi(\lambda, \nu) \\
& y \mapsto \psi(\lambda, \nu)(y, v) \quad \text { is } \quad Y \text {-periodic. }
\end{aligned}
$$

The smallest (positive) eigenvalue $\mu(\lambda, \nu)$ exists for $(\lambda, \mu) \in \mathbb{R}^{+} \times \Lambda$ and we can associate the positive eigenvector $\psi(\lambda, \nu)$. We proceed as in the proof of Proposition 3.1 to show that $\mu(\lambda, \nu)$ and $\psi(\lambda, \nu)$ are twice continuously differentiable. Moreover the partial derivative $\partial_{\lambda} \mu(\lambda, \nu)<0$. Indeed, differentiating (49) with respect to $\lambda$, multiplying by the adjoint function $\psi^{*}(\lambda, \nu)$ and integrating over $Y \times V$ yields

$$
\partial_{\lambda} \mu(\lambda, \nu)=-\frac{\mu(\lambda, \nu)\left(F \psi(\lambda, \nu), \psi^{*}(\lambda, \nu)\right)}{\left((K+\lambda F) \psi(\lambda, \nu), \psi^{*}(\lambda, \nu)\right)},
$$

which is clearly negative as the functions $\psi$ and $\psi^{*}$ are positive. Define now the function $\bar{\mu}(\lambda)=\mu\left(\lambda_{\mu}, \nu_{0}(\lambda)\right)$, where $\nu_{0}$ is the unique point obtained in Proposition 3.1 with $\tilde{K}=K+\lambda F$. We have that $\bar{\mu}(\lambda)$ is decreasing. Indeed, for $\delta \lambda>0$ sufficiently small,

$$
\bar{\mu}(\lambda+\delta \lambda)=\mu\left(\lambda+\delta \lambda, \nu_{0}(\lambda+\delta \lambda)\right) \leq \mu\left(\lambda+\delta \lambda, \nu_{0}(\lambda)\right)
$$

since $\nu_{0}(\lambda+\delta \lambda)$ is a maximum for $\nu_{0} \mapsto \mu\left(\lambda+\delta \lambda, \nu_{0}\right)$ at $\lambda+\delta \lambda$ fixed. Since $\partial_{\lambda} \mu<0$, we also have $\mu\left(\lambda+\delta \lambda, \nu_{0}(\lambda)\right)$ $<\mu\left(\lambda, \nu_{0}(\lambda)\right)=\bar{\mu}(\lambda)$. Thus, $\partial_{\lambda} \bar{\mu}(\lambda)<0$. Now from hypothesis (H3), $\bar{\mu}(0)>1$ since total absorption is greater than scattering. Furthermore, $\bar{\mu}(\lambda) \rightarrow 0$ as $\lambda \rightarrow \infty$ thanks to Theorem 1.4 with $\tilde{K}=K+\lambda F$. By continuity, there exists a unique value of $\lambda_{0}$ such that $\bar{\mu}\left(\lambda_{0}\right)=1$. We denote by $\lambda_{\infty, \nu}$ this value, where $\nu=\nu_{0}\left(\lambda_{0}\right)$. Clearly, equations (26) and (27) are satisfied and $J_{\nu}=\tilde{J}_{\nu}=0$ in (28). When $J=J_{0} \neq 0$, we deduce from the extremality of $\nu$ that $\lambda_{\infty, \nu}>\lambda_{\infty, 0}$. This concludes the proof of Theorem 2.1 .

The author wishes to thank G. Allaire, Y. Capdeboscq, and G. Papanicolaou for their comments and suggestions during the preparation of this work. This work was supported in part by the Direction des Études et Recherches d'Électricité de France and in part by NSF grand DMS-0072008.

\section{REFERENCES}

[1] G. Allaire, Homogenization and two-scale convergence. SIAM J. Math. Anal. 9 (1992) 1482-1518. 
[2] G. Allaire and G. Bal, Homogenization of the criticality spectral equation in neutron transport. ESAIM: M2AN 33 (1999) 721-746.

[3] G. Bal, Couplage d'équations et homogénéisation en transport neutronique, Thèse de Doctorat de l'Université Paris 6 (1997).

[4] G. Bal, Boundary layer analysis in the homogenization of neutron transport equations in a cubic domain. Asymptot. Anal. 20 (1999) 213-239.

[5] G. Bal, First-order Corrector for the Homogenization of the Criticality Eigenvalue Problem in the Even Parity Formulation of the Neutron Transport. SIAM J. Math. Anal. 30 (1999) 1208-1240.

[6] G. Bal, Diffusion Approximation of Radiative Transfer Equations in a Channel. Transport Theory Statist. Phys. (to appear).

[7] P. Benoist, Théorie du coefficient de diffusion des neutrons dans un réseau comportant des cavités, Note CEA-R 2278 (1964).

[8] A. Bensoussan, J.L. Lions and G. Papanicolaou, Asymptotic analysis for periodic structures. North-Holland (1978).

[9] - Boundary Layers and Homogenization of Transport Processes. RIMS, Kyoto Univ. (1979).

[10] J. Bergh and L. Löfström, Interpolation spaces. Springer, New York (1976).

[11] J. Bussac and P. Reuss, Traité de neutronique. Hermann, Paris (1978).

[12] Y. Capdeboscq, Homogenization of a diffusion equation with drift. C. R. Acad. Sci. Paris Sér. I Math. 327 (1998) 807-812.

[13] — Homogenization of a Neutronic Critical Diffusion Problem with Drift. Proc. Roy Soc. Edinburgh Sect. A (accepted).

[14] F. Chatelin, Spectral approximation of linear operators. Academic Press, Comp. Sci. Appl. Math. (1983).

[15] R. Dautray and J.L. Lions, Mathematical analysis and numerical methods for Science and Technology, Vol. 6. Springer Verlag, Berlin (1993).

[16] V. Deniz, The theory of neutron leakage in reactor lattices, in Handbook of nuclear reactor calculations, Vol. II, edited by Y. Ronen (1968) 409-508.

[17] J. Garnier, Homogenization in a periodic and time dependent potential. SIAM J. Appl. Math. 57 (1997) 95-111.

[18] F. Golse, P.-L. Lions, B. Perthame and R. Sentis, Regularity of the moments of the solution of a transport equation. J. Funct. Anal. 76 (1988) 110-125.

[19] T. Kato, Perturbation theory for linear operators. Springer Verlag, Berlin (1976).

[20] M.L. Kleptsyna and A.L. Piatnitski, On large deviation asymptotics for homgenized SDE with a small diffusion. Probab. Theory Appl. (submitted).

[21] S. Kozlov, Reductibility of quasiperiodic differential operators and averaging. Trans. Moscow Math. Soc. 2 (1984) 101-136.

[22] E.W. Larsen, Neutron transport and diffusion in inhomogeneous media. I. J. Math. Phys. 16 (1975) 1421-1427.

[23] — Neutron transport and diffusion in inhomogeneous media. II. Nuclear Sci. Engrg. 60 (1976) 357-368.

[24] E.W. Larsen and J.B. Keller, Asymptotic solution of neutron transport problems for small mean free paths. J. Math. Phys. 15 (1974) 75-81.

[25] E.W. Larsen and M. Williams, Neutron Drift in Heterogeneous Media. Nuclear Sci. Engrg. 65 (1978) 290-302.

[26] M. Mokhtar-Kharroubi, Mathematical Topics in Neutron Transport Theory. World Scientific, Singapore (1997).

[27] J. Planchard, Méthodes mathématiques en neutronique, Collection de la Direction des Études et Recherches d'EDF. Eyrolles (1995).

[28] L. Ryzhik, G. Papanicolaou and J.B. Keller, Transport equations for elastic and other waves in random media. Wave Motion 24 (1996) 327-370.

[29] R. Sentis, Study of the corrector of the eigenvalue of a transport operator. SIAM J. Math. Anal. 16 (1985) 151-166.

[30] M. Struwe, Variational methods: Applications to nonlinear partial differential equations and Hamiltonian systems. Springer, Berlin (1990). 